\title{
Cerebral Fat Embolism Syndrome from Penetrating Trauma: A Rare Cause-and-Effect
}

\author{
Stephen J. Gleich*, James D. Hannon ${ }^{*}$ \\ Department of Anesthesiology, Mayo Clinic, Rochester, USA. \\ Email: gleich.stephen@mayo.edu, ${ }^{\#}$ hannon.james@mayo.edu \\ Received January $26^{\text {th }}, 2013$; revised March $1^{\text {st }}, 2012$; accepted April $1^{\text {st }}, 2013$ \\ Copyright (C) 2013 Stephen J. Gleich, James D. Hannon. This is an open access article distributed under the Creative Commons At- \\ tribution License, which permits unrestricted use, distribution, and reproduction in any medium, provided the original work is prop- \\ erly cited.
}

\begin{abstract}
A 42 year-old male sustained an accidental rifle gunshot wound to his left foot, resulting in fracture deformities of the calcaneus, navicular, cuneiform, $1^{\text {st }}$ and $2^{\text {nd }}$ metatarsal bases, and talus. As he was transported to our trauma center, he developed progressive encephalopathy. Urgent external fixator placement under general anesthesia was postponed due to his encephalopathy of unknown etiology. Brain magnetic resonance imaging demonstrated a "starfield" pattern of infarcts, consistent with cerebral fat embolism syndrome. Subsequently, he underwent uneventful general anesthesia. The patient was managed supportively and continued to have persistent neurologic dysfunction two months after injury.
\end{abstract}

Keywords: Penetrating Trauma; Cerebral Fat Embolism Syndrome

\section{Introduction}

Fat embolism syndrome (FES) is a potentially devastateing complication of musculoskeletal trauma that has historically been underdiagnosed [1]. Lack of awareness of the associated signs and symptoms and the frequent masking of diagnostic criteria by multiple injuries in trauma patients hinders the diagnosis. In addition, the expected classical presentation of confusion, dyspnea, and petechia is not always observed. Clinical criteria were established for the diagnosis of FES by Gurd and Wilson in 1970 [2]. Documentation of at least one major and four minor criteria plus fat macroglobulinemia is generally considered adequate [3]. The syndrome occurs more commonly in major orthopedic surgery and closed long-bone fractures via blunt trauma, with a higher incidence observed in closed femur fractures [4]. Penetrating trauma is a rare cause of FES, probably related to the lower probability of long bone fractures compared to blunt trauma. In this report, we present the case of a healthy gentleman who developed isolated cerebral FES after penetrating trauma from an accidental gunshot wound to the foot.

\footnotetext{
${ }^{*}$ Contribution: Data collection, data analysis, and manuscript preparation.

${ }^{\#}$ Corresponding author.
}

\section{Case Description}

An otherwise healthy 42 year-old male sustained an accidental, close-range, gunshot wound to his left foot from a 270-caliber hunting rifle while on a family hunting trip. He was initially evaluated at a local hospital and found to have a normal mental status. Over the course of approximately 6 hours (throughout the initial evaluation at the referring hospital and during transport), he received 7 milligrams of hydromorphone for pain control along with antibiotic and antiemetic therapy. He was transferred via ground ambulance to our trauma center for definitive orthopedic surgical management. During the transport, the patient's mental status progressively declined but he was apparently still able to respond to questioning appropriately.

Upon arrival at our facility, orthopedic trauma evaluation was performed. At this point, his neurological status had further deteriorated and he was unresponsive with only brief, nonpurposeful movement to painful stimulus. He demonstrated horizontal roving eye movements, occasional irregular arm jerks, right ankle sustained clonus, and extensor plantar response. Examination of the left lower extremity revealed a small gun shot entrance wound medial to the Achilles tendon with an exit wound on the plantar aspect of the foot near the heads of the first and second metatarsals. Significant pedal edema was 
observed with intact pedal pulses. His initial foot X-ray (Figure 1) showed metallic shot fragments with traumatic fracture deformities of the calcaneus, navicular, medial cuneiform, $1^{\text {st }}$ and $2^{\text {nd }}$ metatarsal bases, and talus. Laboratory studies were obtained and remarkable for hemoglobin of $12.8 \mathrm{~g} / \mathrm{dL}$, platelet count of $249 \times 10^{9} / \mathrm{L}$, serum white blood cell count of $21 \times 10^{9} / \mathrm{L}$, glucose 193 $\mathrm{mg} / \mathrm{dL}$, creatine kinase $667 \mathrm{U} / \mathrm{L}$, electrolytes and liver function testes were within normal limits.

Given the patient's altered mental status, he received a standard dose of naloxone in the Emergency Department (ED), without improvement in his mental status. He was brought to the operating room (OR) for left foot irrigation and debridement with external fixator placement. Prior to induction, he was further evaluated by the anesthesiologist and remained obtunded. Due to his persistent significant cognitive decline of unknown etiology, surgery was canceled.

The patient returned to the ED and neurology consultation was obtained. A lumbar puncture was performed with normal opening pressure, and normal cell counts, protein, and glucose. An emergent electroencephalogram showed generalized diffuse slowing without epileptogenic discharges or seizure activity, consistent with encephalopathy. Computed tomography scanning (CT) of the head showed no acute findings. The patient was admitted to the intensive care unit with no obvious cause for his coma. Shortly after admission he required endotracheal intubation due to inability to protect his airway. Magnetic resonance imaging (MRI) was delayed until the second hospital day due to concerns of metallic bullet fragements abutting the doraslis pedis artery. Once performed, brain MRI demonstrated innumerable tiny infarcts throughout the cerebral hemispheres, cerebellum, and brainstem, consistent with cerebral fat embolism syndrome (Figure 2).
Supportive care was continued throughout his hospital course. A transthoracic echocardiogram showed a small right to left shunt, likely from intrapulmonary shunting, without evidence of a definitive patent foramen ovale (PFO). He developed thrombocytopenia (platelet count of $88 \times 10^{9} / \mathrm{L}$ ) and unexplained anemia (hemoglobin 6.6 $\mathrm{g} / \mathrm{dL}$ ), with nadirs on the forth and fifth hospital days, respectively. Following very slight improvement in his neurologic exam, he underwent external fixator placement on the fourth hospital day under general anesthesia, which he tolerated well. Throughout his hospitalization, he required only minimal ventilator settings without pulmonary infiltrates or dysfunction. No petechial rash was observed. The patient had intermittent tachycardia and pyrexia throughout his hospital course.

The patient had no substantial neurological improvement and underwent bedside percutaneous tracheostomy on the sixth hospital day. Repeat MRI on the eighth hospital day showed expected progression of cerebral fat embolism syndrome, with confluence of the previous tiny infarcts. Percutaneous endoscopic gastrostomy tube placement was performed on hospital day number 16 . The patient was discharged on hospital day number 19 to a long-term rehab care center. At discharge, the patient's neurological exam was only minimally improved, with spontaneous eye opening, blinking to threat, however, he did not fixate nor track. He continued to have spontaneous trismus, extensor posturing, and increase tone throughout.

Approximately 2 months after his injury, the patient had slowly improving cognitive function, followed simple commands, initiated speech, and was more aware of his surroundings.

\section{Discussion}

This report details the rare case of profound neurologic

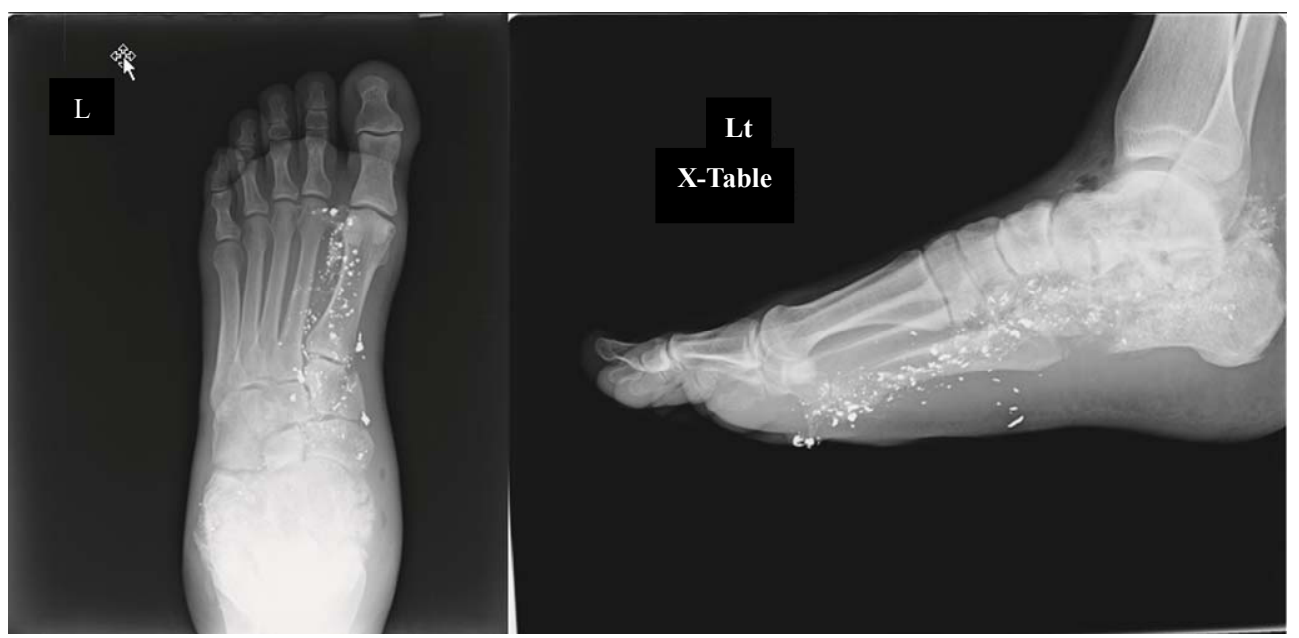

Figure 1. Initial foot X-ray showing metallic shot fragments with traumatic fracture deformities of the calcaneus, navicular, cuneiform, and first and second metatarsals. 


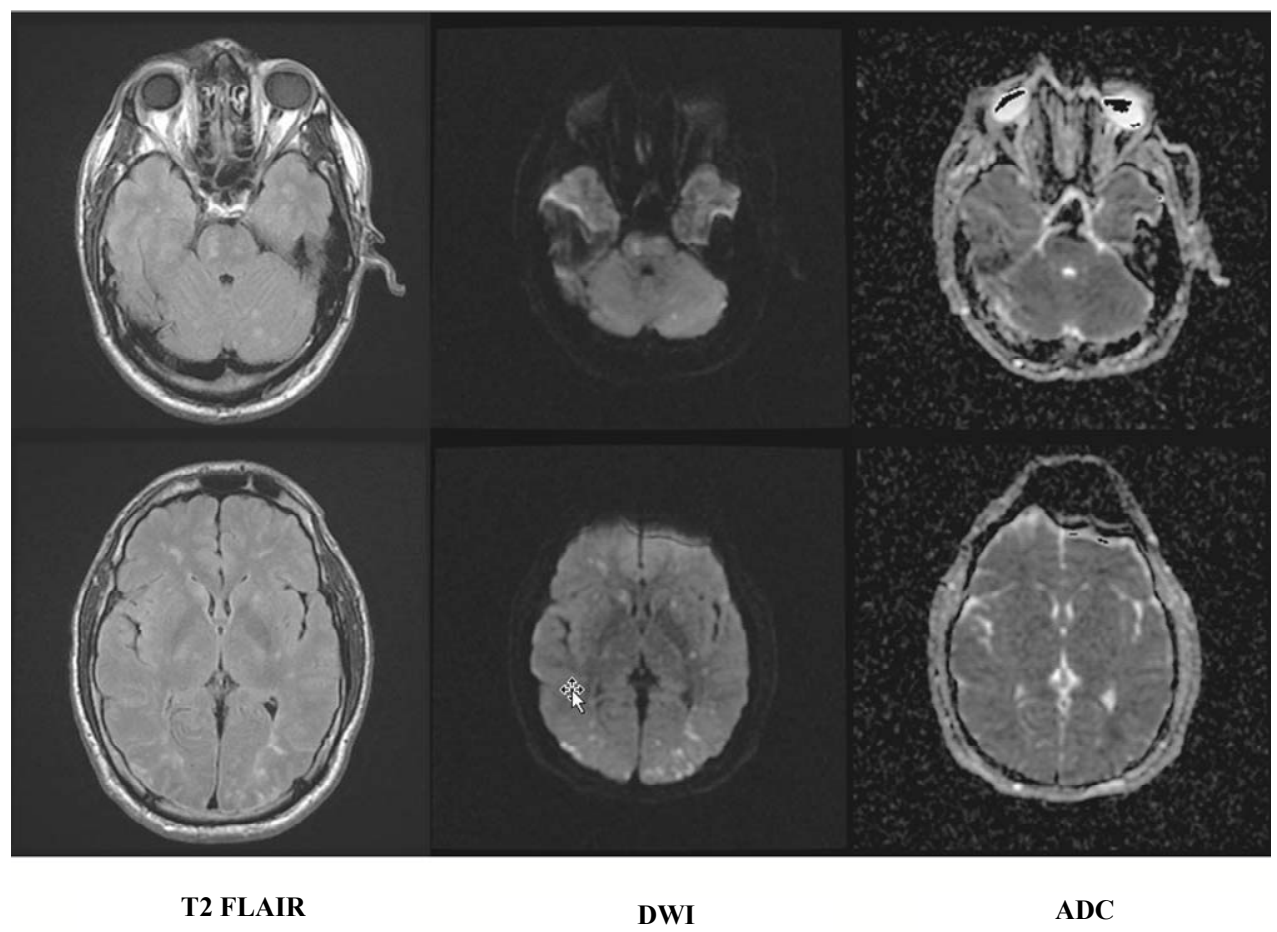

Figure 2. MRI showing innumerable tiny infarcts throughout the cerebral hemispheres, cerebellum, and brainstem.

deterioration from cerebral FES in a patient who sustained penetrating trauma to multiple small bones in the foot. Major criteria, as proposed by Gurd [2] for the diagnosis of FES include: petechial rash, respiratory symptoms with chest X-ray findings, and neurologic deterioration unrelated to head injury. Additional minor criteria include: tachycardia, fever, anemia, thrombocytopenia, and elevated erythrocyte sedimentation rate. The reported incidence of FES is widely variable and ranges from $1 \%$ - $2 \%$, when studied retrospectively, to $11 \%$ - $19 \%$ when examined prospectively [5].

Given the absence of long bone skeletal trauma in our patient, the initial differential diagnosis of his cognitive decline was broad and included opioid overdose, hypoxic-ischemic encephalopathy, nonconvulsive status epilepticus, or a rare infectious or inflammatory encephalopathy. The diagnosis of FES was made more difficult by the absence of pulmonary or cutaneous manifestations. Our patient still satisfied multiple criteria for FES, including intermittent tachycardia, pyrexia, and otherwise unexplained anemia and thrombocytopenia.

Reported cases of cerebral fat embolism syndrome are most commonly due to musculoskeletal large bone blunt trauma [6]. Utilizing a series of 50 autopsy cases, fat embolism was identified in $82 \%$ of decedents of traumatic injuries, with the vast majority (89\%) as victims of blunt trauma [1]. Penetrating trauma is a rare cause of fat embolism with very few cases reported.

One case of cerebral fat embolism, similar to ours, occurred in a 19 year-old male who sustained gunshot wounds to the right hand and right knee [7]. His injuries included open proximal phalynx fracture of the right ring finger and an open right lateral femoral condyle fracture. He developed generalized seizure activity upon arrival to the hospital, with obtundation, and decerebrate posturing. An MRI showed hyperintense dot-like lesions, consistent with a "starfield" pattern. A transthoracic echocardiogram was normal. He was managed supportively and 10 weeks later, his neurological status had normalized.

The pathophysiology of FES has been described by two mechanisms. The first mechanism involves physical embolization of intramedullary fat into the venous system following trauma [8]. Once the fat microdroplets have reached the pulmonary vasculature, they can be sequestered by the lungs resulting in pulmonary dysfunction, or gain access to the systemic circulation via intracardiac or intrapulmonary shunt and cause neurologic changes [5]. Surprisingly, our patient only had a small intrapulmonary shunt when evaluated with echocardiography, without definitive evidence of a PFO. Our patient developed profound isolated neurologic manifestations very quickly after his injury. Presumably, the force of the high-velocity gunshot wound to his foot resulted in an immediate large embolic shower, which efficiently reached the arterial circulation assisted by a small shunt. It is also thought that fat microdroplets can travel through the pulmonary vasculature without sequestration, presumably due to their very small size or deformability, resulting directly in systemic manifestations without pulmonary involvement, further resulting in isolated neur- 
ologic manifestations as seen in our patient [9].

The second pathophysiologic mechanism is biochemical in nature $[5,8]$. Traumatic injury causes plasma lipase levels to rise, increasing the release of free fatty acids and resulting in altered fat homeostasis. Consequently, fat droplets are further formed and subsequently sequestered in the microvasculature [10]. An inflammatory reaction in the microvasculature is proposed to ensue from free fatty acid release, resulting in intensified end-organ dysfunction. Interestingly, other than isolated cerebral injury, our patient showed no other signs of endorgan dysfunction, implying that an exaggerated inflammatory reaction was limited to the cerebral injury. Although the exact mechanism of FES is unknown in our patient, some combination of both mechanisms in the pathophysiology likely occurred.

Magnetic resonance imaging played a key role in the diagnosis of cerebral FES in our patient and has shown to be a sensitive tool to detect embolic infarcts [11]. Unfortunately, CT scanning is usually unrevealing in patients with cerebral FES, but serves to exclude other causes of altered mental status [12]. The classic MRI findings include a "starfield" pattern of multiple, diffuse, tiny hyperintense lesions across the cerebral white matter with restricted diffusion [7].

The treatment of FES remains supportive [5]. Early fixation of fractures has been shown to decrease the incidence of fat embolism syndrome [13]. Our patient underwent fracture reduction and external fixator placement on the fourth hospital day. Given that he had severe clinical manifestations of cerebral FES upon arrival, the benefit of more expeditious fixation is questionable. Once FES has developed, many interventions including aspirin, heparin, and dextrans have shown no benefit. The data on corticosteroids is mixed, with several small studies showing improved gas exchange in FES patients with pulmonary dysfunction $[14,15]$ and others showing no benefit [16]. These studies are further confounded by variable dosage and timing of corticosteroid administration. There is no known intervention to improve neurologic outcome in patients with cerebral FES.

Due to the rarity of cerebral fat embolism, clinical outcomes are also largely unknown. Isolated case reports range from complete recovery $[17,18]$ to mild residual cognitive impairments $[12,19]$ to progressive intracranial hypertension and brain death [20]. In several case reports, some neurologic improvement was noted by the time of discharge. Although our patient had minimal improvement at discharge, he began showing signs of neurologic recovery within 2 months of injury.

In conclusion, fat embolism is common in traumatically injured patients. Isolated cerebral FES from penetrating trauma is a rare presentation with largely unknown outcomes. Anesthesia providers and trauma prac- titioners are encouraged to remain vigilant for FES in the setting of neurologic or pulmonary dysfunction associated with traumatic injury.

\section{REFERENCES}

[1] E. A. Eriksson, D. C. Pellegrini, W. E. Vanderkolk, C. T. Minshall, S. M. Fakhry and S. D. Cohle, "Incidence of Pulmonary Fat Embolism at Autopsy: An Undiagnosed Epidemic," Journal of Trauma, Vol. 71, No. 2, 2011, pp. 312-315. doi:10.1097/TA.0b013e3182208280

[2] A. R. Gurd, "Fat Embolism: An Aid to Diagnosis," Journal of Bone and Joint Surgery, Vol. 52, No. 4, 1970, pp. 732-737.

[3] A. R. Gurd and R. I. Wilson, "The Fat Embolism Syndrome," Journal of Bone and Joint Surgery, Vol. 56B, No. 3, 1974, pp. 409-416.

[4] S. Akhtar, "Fat Embolism," Anesthesiology Clinics, Vol. 27, No. 3, 2009, pp. 533-550. doi:10.1016/j.anclin.2009.07.018

[5] A. Mellor and N. Soni, "Fat Embolism," Anaesthesia, Vol. 56, No. 2, 2001, pp. 145-154. doi:10.1046/j.1365-2044.2001.01724.x

[6] C. W. Ryu, D. H. Lee, T. K. Kim, S. J. Kim, H. S. Kim, J. H. Lee, C. G. Choi and D. C. Suh, "Cerebral Fat Embolism: Diffusion-Weighted Magnetic Resonance Imaging Findings," Acta Radiologica, Vol. 46, No. 5, 2005, pp. 528-533. doi:10.1080/02841850510021481

[7] A. Aravapalli, J. Fox and C. Lazaridis, "Cerebral Fat Embolism and the 'Starfield' Pattern: A Case Report," Cases Journal, Vol. 2, No. 1, 2009, p. 212. doi:10.1186/1757-1626-2-212

[8] K. Taviloglu and H. Yanar, "Fat Embolism Syndrome," Surgery Today, Vol. 37, No. 1, 2007, pp. 5-8. doi:10.1007/s00595-006-3307-5

[9] R. J. Byrick, J. B. Mullen, C. D. Mazer and C. B. Guest, "Transpulmonary Systemic Fat Embolism. Studies in Mongrel Dogs after Cemented Arthroplasty," American Journal of Respiratory and Critical Care Medicine, Vol. 150, No. 5, 1994, pp. 1416-1422.

[10] F. Nastanski, W. I. Gordon and M. E. Lekawa, "Posttraumatic Paradoxical Fat Embolism to the Brain: A Case Report," Journal of Trauma, Vol. 58, No. 2, 2005, pp. 372-374. doi:10.1097/01.TA.0000108996.10785.0B

[11] H. Satoh, K. Kurisu, M. Ohtani, K. Arita, S. Okabayashi, T. Nakahara, K. Migita, K. Iida, K. Kuroki and N. Ohbayashi, "Cerebral Fat Embolism Studied by Magnetic Resonance Imaging, Transcranial Doppler Sonography, and Single Photon Emission Computed Tomography: Case Report," Journal of Trauma, Vol. 43, No. 2, 1997, pp. 345-348. doi:10.1097/00005373-199708000-00023

[12] M. E. Finlay and M. D. Benson, "Case Report: Magnetic Resonance Imaging in Cerebral Fat Embolism," Clinical Radiology, Vol. 51, No. 6, 1996, pp. 445-446. doi:10.1016/S0009-9260(96)80169-3

[13] S. J. Pinney, J. F. Keating and R. N. Meek, "Fat Embolism Syndrome in Isolated Femoral Fractures: Does Timing of Nailing Influence Incidence?" Injury, Vol. 29, No. 
2, 1998, pp. 131-133. doi:10.1016/S0020-1383(97)00154-X

[14] J. Kallenbach, M. Lewis, M. Zaltzman, C. Feldman, A. Orford and S. Zwi, “'Low-Dose Corticosteroid Prophylaxis against Fat Embolism," Journal of Trauma, Vol. 27, No. 10, 1987, pp. 1173-1176. doi:10.1097/00005373-198710000-00013

[15] B. G. Lindeque, H. S. Schoeman, G. F. Dommisse, M. C. Boeyens and A. L. Vlok, "Fat Embolism and the Fat Embolism Syndrome. A Double-Blind Therapeutic Study," Journal of Bone and Joint Surgery, Vol. 69, No. 1, 1987, pp. 128-131.

[16] R. J. Byrick, J. B. Mullen, P. Y. Wong, J. C. Kay, D. Wigglesworth and R. J. Doran, "Prostanoid Production and Pulmonary Hypertension after Fat Embolism Are Not Modified by Methylprednisolone," Canadian Journal of Anaesthesia, Vol. 38, No. 5, 1991, pp. 660-667. doi:10.1007/BF03008205

[17] H. Chrysikopoulos, V. Maniatis, J. Pappas, P. Filalithis, C. Gogali and D. Sfyras, "Case Report: Post-Traumatic
Cerebral Fat Embolism: CT and MR findings. Report of Two Cases and Review of the Literature," Clinical Radiology, Vol. 51, No. 10, 1996, pp. 728-732. doi:10.1016/S0009-9260(96)80248-0

[18] M. Scopa, M. Magatti and P. Rossitto, "Neurologic Symptoms in Fat Embolism Syndrome: Case Report," Journal of Trauma, Vol. 36, No. 6, 1994, pp. 906-908. doi:10.1097/00005373-199406000-00030

[19] G. Manousakis, D. Y. Han and M. Backonja, "Cognitive Outcome of Cerebral Fat Embolism," Journal of Stroke and Cerebrovascular Diseases, Vol. 21, No. 8, 2012, pp. $1-3$.

http://www.ncbi.nlm.nih.gov/pubmed?term=Manousakis $\% 20 \mathrm{G} \% 20$ Cognitive\%20Outcome

[20] E. A. Eriksson, S. E. Schultz, S. D. Cohle and K. W. Post, "Cerebral Fat Embolism without Intracardiac Shunt: A Novel Presentation," Journal of Emergencies, Trauma, and Shock, Vol. 4, No. 2, 2011, pp. 309-312. doi:10.4103/0974-2700.82233

\begin{abstract}
Abbreviations
DWI: Diffusion weighted MRI,

ADC: Apparent diffusion coefficient,

IRB: This study is considered a "no risk" case and is exempt from IRB review according to the Mayo Foundation IRB.
\end{abstract}

